ఠ

\title{
Anti-colorectal cancer effect of interleukin-2 and interferon- $\beta$ fusion gene driven by carcinoembryonic antigen promoter
}

This article was published in the following Dove Press journal:

OncoTargets and Therapy

30 May 2016

Number of times this article has been viewed

\section{Yan Wang \\ Mengchun Wang \\ Yan Li}

Department of Gastroenterology, Shengjing Hospital of China Medical University, Shenyang, People's Republic of China
Correspondence: Yan Li Department of Gastroenterology, Shengjing Hospital of China Medical University, 36 Sanhao Street, Shenyang I I0004, People's Republic of China Tel/fax +86242389 6615

Email wangy9@sj-hospital.org

\begin{abstract}
This study was designed to investigate the antitumor effects of combined interleukin-2/interferon- $\beta$-based gene therapy in colorectal cancer. Transfection of the fusion gene expression plasmid induced significant apoptosis of Lovo cells. Additionally, the fusion gene exhibited strong inhibitory activity against tumor growth and apoptosis when being injected into the nude mice implanted with human colon cancer cells. Furthermore, the tail-vein injection showed a more notable effect than direct injection into tumor. These results suggest that the combined interleukin-2/interferon- $\beta$-based gene therapy with the carcinoembryonic antigen promoter might be an effective antitumor strategy.
\end{abstract}

Keywords: apoptosis, interferon- $\beta$, interleukin- 2 , antitumor, combined gene therapy

\section{Introduction}

Colorectal cancer (CRC), one of the most prevalent malignancies globally, caused approximately 1.4 million new cases and resulted in 694,000 CRC-related deaths in $2012 .{ }^{1}$ Moreover, metastases were frequently detected in $>40 \%$ of patients with $\mathrm{CRC},{ }^{2}$ which led to the inappropriate application of surgical therapy and frequent recurrence after radiotherapy and chemotherapy. ${ }^{3}$ Thus, there is an urgent need to develop novel treatment strategies.

To our knowledge, several studies have demonstrated that the interaction among environmental carcinogens, the host immune system, and genetic alterations plays important roles in the progression of $\mathrm{CRC}$, which ultimately causes the uncontrolled cell growth. ${ }^{4}$ Thus, therapy with immune-associated genes shows great potential advantages in the treatment of cancer. ${ }^{5,6}$ Both interferon- $\beta$ (IFN- $\beta$ ) and interleukin- 2 (IL-2) are important cytokines and play multiple roles in host immune system. They are also shown to display remarkable antitumor potential. ${ }^{7,8}$ Notably, the cytokines have been already used in clinic to treat some tumors and other diseases., ${ }^{9,10}$ The IL-2 gene when transfected into the $\mathrm{C}-26$ colon cancer cells significantly inhibited tumor growth in $76 \%$ of mice $7-10$ days post-transfection. ${ }^{11,12}$ Gunji et al have also found that the survival rate of mice increased significantly after the IL-2 gene was transfected into C-26 colon cancer cells. ${ }^{13}$ Qin et al have shown that tumor formation was found in only $1 \%$ of mice, but tumor suppression was observed in most of the mice after IFN- $\beta$ gene therapy by means of replication-defective retroviral vector. ${ }^{14}$ However, the efficacy of single cytokine on tumor in clinical trials has been limited. The benefits of these combined cytokines are encouraging. ${ }^{15}$ A former study has demonstrated that IFN and IL-2 induce lymphokine-activated killer activity synergistically, further enhancing the 
sensitivity of tumor cells to killer cells. ${ }^{16}$ Another previous report has demonstrated the synergistic antitumor effect of IL-2 and IFN- $\beta .{ }^{17}$

Although many gene therapy methods have been used in clinical trials, such as cytokines and herpes simplex virus thymidine kinase, and have made some progress in the treatment of cancer, ${ }^{18}$ there remains several shortcomings. Among them, the specificity and efficiency of gene expression in tumor cells and safety to normal cells is a major limitation. As we all know, carcinoembryonic antigen (CEA), one of the earliest identified tumor-associated antigens, is found in many gland tumors, especially in the tumors of the digestive system. ${ }^{19}$ More than 90\% of CRC cells express CEA. Moreover, gene expression regulated by a tumor-specific CEA can improve the effect of gene therapy and reduce the side effects of treatment. ${ }^{20}$ Thus, we aimed to investigate CEA promoter activities and to achieve tumor-specific transgene expression using the CEA promoter.

In this study, we constructed expression plasmid pcDNA3.1A-IL-2/IFN- $\beta$ as well as pcDNA3.1A-CEA-IL-2/ IFN- $\beta$, and demonstrated synergistic antitumor effects of combined IL-2/IFN- $\beta$-based gene therapy in a murine CRC model. The effects of combined IL-2/IFN- $\beta$-based gene therapy were examined by flow cytometry, hematoxylin and eosin (HE) staining, and transmission electron microscopy.

\section{Materials and methods}

\section{Cell lines and experimental animals}

Colon cancer tissue samples were obtained from the first affiliated hospital of China Medical University. Lovo and HT-29 cell lines were cultured in Dulbecco's Modified Eagle's Medium (Sigma Chemical Co., St Louis, MO, USA), supplemented with $10 \%$ fetal bovine serum and antibiotics at $37^{\circ} \mathrm{C}$ in $5 \% \mathrm{CO}_{2}$. The use of human cells was approved by the Ethics Committee of the First Affiliated Hospital of China Medical University.

A total of 30 male BALB/C nude mice, weighing 18-22 g (4-6 weeks old), were purchased from Experimental Animal Center of China Medical University. All animal experiments in the present study were performed according to the guidelines for the care and use of laboratory animals, with Animal Ethics Committee approval from the First Affiliated Hospital of China Medical University.

\section{Plasmids construction and transfection}

The open reading frame (ORF) of human IL-2 (461 bp) was amplified using the cDNA reversely transcripted from normal human peripheral blood lymphocytes by the primers of IL-2: forward (5'-TCGAATTggatccCTACCATGTACAGGATG-3') and reverse (5'-GCAGGAATgaattcCAGTGTTGAGATG
ATG-3'). The ORF of IFN- $\beta$ (563 bp) was amplified from plasmid pCMV (Hu beta) (RIKEN Institute, Wako, Japan) using the primers of IFN- $\beta$ : forward $\left(5^{\prime}\right.$-GATCCGATTgcgg ccgcATGACCAACAAG-3') and reverse (5'-GCTAGGctcg agTCAGTTTCGGAGGTA-3'). The ORF of IL-2 and IFN- $\beta$ was linked using linker primer (gaattctGGTGGCTCTGGA TCTGGCGGTTCTGGATCTGGTGGCTCTGgcggccgc) to generate the fragment IL-2/IFN- $\beta$, which was cloned into the XhoI and BamHI restriction sites of pEGFP-C1 (Clotech, Mountain View, CA, USA) and pcDNA 3.1A (Invitrogen, Carlsbad, CA, USA) to generate the plasmid pEGFP-IL-2/ IFN- $\beta$ and pcDNA3.1A-IL-2/IFN- $\beta$, respectively.

The CEA promoter fragment (504 bp) was amplified from colon cancer tissue DNA using the primers of CEA: forward (5'-ATGaagcttTTTCTAGCCCCCAGAGCCACC-3') and reverse (5'-GAGGGaagcttCATGGTCTCTGCTGTCT GC-3'). The CEA promoter fragment was then inserted into the Hind III restriction sites of pcDNA3.1A-IL-2/IFN- $\beta$ to generate the plasmid pcDNA3.1A-CEA-IL-2/IFN- $\beta$. All the plasmids were confirmed by sequencing. Transient and stable transfections were performed using lipofectamine 2000 (Invitrogen) according to the product manual. Cells with stable expression of plasmid were selected using G418 (Roche, Basel, Switzerland) at a concentration of $400 \mu \mathrm{g} / \mathrm{mL}$ at 2 weeks and maintained in medium containing $200 \mu \mathrm{g} / \mathrm{mL}$ G418 for 6-8 weeks.

\section{DNA ladder electrophoresis}

The specimen was homogenized and suspended in buffer (10 mmol/L Tris- $\mathrm{HCl}$ [pH 7.5], $0.1 \mathrm{~mol} / \mathrm{L} \mathrm{NaCl}$, and $1 \mathrm{mmol} / \mathrm{L}$ ethylenediaminetetraacetic acid [EDTA]), followed by addition of $1 \mathrm{~mL}$ of $10 \%$ sodium dodecyl sulfate, and incubation with proteinase $\mathrm{K}$ at $50^{\circ} \mathrm{C}$ overnight. DNA was extracted twice with phenol and twice with phenol/chloroform (1/1); the upper layer was collected, and $5 \mathrm{~mol} / \mathrm{L} \mathrm{NaCl}$ was added at a $1: 50(\mathrm{v} / \mathrm{v})$ ratio and then precipitated at $-20^{\circ} \mathrm{C}$ in absolute ethanol overnight. DNA was pelleted by centrifugation at $3,000 \mathrm{rpm}$ at $4^{\circ} \mathrm{C}$ for 10 minutes, washed with $70 \%$ ethanol, and dried. DNA was re-suspended in buffer $(10 \mathrm{mmol} / \mathrm{L}$ Tris- $\mathrm{HCl}$ and $1 \mathrm{mM}$ EDTA). Samples were incubated with $0.1 \mathrm{mg} / \mathrm{mL}$ DNAse-free RNAse 21 at $37^{\circ} \mathrm{C}$ for 30 minutes. Then, $0.25 \%$ xylene cyanol in $40 \%$ sucrose and $4 \mu \mathrm{L}$ of $0.25 \%$ bromophenol blue were added to samples at a ratio of 1:5 (v/v). Electrophoresis was performed in 1.6\% agarose gels at $50 \mathrm{~V}$, and DNA was visualized with ethidium bromide.

\section{Indirect immunofluorescence microscopy}

In brief, Lovo cells were transfected with indicated plasmid and then fixed in $4 \%$ paraformaldehyde. Cells were incubated with 
rabbit anti-IL- 2 or anti-IFN- $\beta$ at $37^{\circ} \mathrm{C}$ for 1 hour, followed by incubation with tetramethyl rhodamine isocyanate-conjugated goat anti-rabbit IgG (Beijing Zhongshan Biotechnology Co., Beijing, People's Republic of China). Afterward, cells were washed extensively with phosphate-buffered saline (PBS). Subsequently, the sections were mounted with 50\% glycerinum. As a negative control, sections were incubated with PBS instead of primary antibodies. The cells positive for IL-2 and IFN- $\beta$ staining were counted using Image-Pro Plus 6.0 software. Samples were analyzed by fluorescence microscopy (L-120; Nikon, Tokyo, Japan).

\section{Flow cytometry}

After digestion with trypsin, cells were fixed with $70 \%$ ethanol at $4{ }^{\circ} \mathrm{C}$ for 24 hours. Cells were then incubated with $1 \mathrm{mg} / \mathrm{mL}$ RNase A for 30 minutes to 1 hour, followed by propidium iodide treatment for 2 hours in the dark. Then, flow cytometry assays were carried out to detect cell cycle at $480 \mathrm{~nm}$. Samples were processed and screened using a fluorescence-activated cell sorter.

\section{Western blotting}

Total proteins were extracted from cultured cells of each group with $1 \mathrm{mM}$ phenylmethanesulfonyl fluoride in $1 \mathrm{~mL}$ ice-cold radioimmunoprecipitation assay buffer, and then added to EDTA-free protease inhibitor cocktail. The protein concentrations were determined by the bicinchoninic acid protein assay. An identical amount of protein $(30 \mu \mathrm{g})$ from each group was loaded onto a $10 \%$ sodium dodecyl sulfatepolyacrylamide gel and electrophoresed at $100 \mathrm{~V}$. Then, the proteins were transferred to polyvinylidene difluoride membranes at $200 \mathrm{~mA}$ for 2 hours at room temperature in a Bio-Rad TransBlot apparatus, followed by blocking of nonspecific binding sites with 5\% nonfat milk in Trisbuffered saline/Tween 20 (TBST). Then, each membrane was incubated with IL-2 (Santa Cruz Biotechnology, Dallas, TX, USA) or IFN- $\beta$ primary antibodies at $4^{\circ} \mathrm{C}$ overnight (Santa Cruz Biotechnology), according to product manuals. After being washed with TBST, the membranes were incubated with alkaline phosphatase-conjugated goat anti-rabbit IgG (Beijing Zhongshan Biotechnology Co.). Then, the membranes were washed five times with TBST. Protein bands specific to the antibody were developed by 5-bromo-4-chloro-3-indoyl-phosphate and nitro-blue tetrazolium.

\section{Enzyme-linked immunosorbent assay}

IL-2 and IFN- $\beta$ quantitative enzyme-linked immunosorbent assay (ELISA) kits were bought from male Science and Technology Industrial Co., LTD (Shanghai, People's Republic of China), and the ELISAs were performed according to product manuals. Briefly, each well was incubated with primary antibodies of IL- 2 and IFN- $\beta$ at $4{ }^{\circ} \mathrm{C}$ overnight. The 96-well plate was washed four times with PBS, and then further incubated with $100 \mu \mathrm{L}$ of substrate solution for 10 minutes at $37^{\circ} \mathrm{C}$ in the dark. Additionally, stop solution was added. Absorbance was read on a microplate reader at $492 \mathrm{~nm}$. The control wells were incubated in PBS without primary antibodies.

\section{In vivo murine colon carcinoma model and interventions}

Nude mice were injected with $1 \times 10^{7}$ Lovo cells, and tumor formation was observed at the tenth day after the administration of Lovo cells. When primary tumors reached $100 \mathrm{~mm}^{3}$ in volume, mice were treated with gene therapy. The tumor volume of five mice was $<100 \mathrm{~mm}^{3}$. Treatment was carried out on the rest 25 tumor-transplanted mice which were divided into five groups ( $\mathrm{N}=5$ /group): Group 1, mice were treated with Dulbecco's Modified Eagle's Medium by intratumor injection as control group; Group 2, mice were treated with the complex containing $20 \mu \mathrm{L}$ liposomes and $8 \mu \mathrm{g}$ pcDNA3.1A-IL-2/IFN- $\beta$ plasmid by intratumor injection; Group 3, mice were treated with the complex containing $20 \mu \mathrm{L}$ liposomes and $8 \mu \mathrm{g}$ pcDNA3.1A-IL-2/IFN- $\beta$ plasmid by tail intravenous injection; Group 4, mice were treated with the complex containing $20 \mu \mathrm{L}$ liposomes and $8 \mu \mathrm{g}$ pcDNA3.1A-CEA-IL-2/IFN- $\beta$ plasmid by intratumor injection; Group 5, mice were treated with the complex containing $20 \mu \mathrm{L}$ liposomes and $8 \mu \mathrm{g}$ pcDNA3.1A-CEAIL-2/IFN- $\beta$ plasmid by tail intravenous injection. Animals were kept in individual cages by groups. After 5 days, the mice were injected for a second time, and the size of the tumor was monitored for 14 days. Animals were weighed and recorded. Tumor dimensions were measured with calipers, and the tumor volume was calculated according to the formula: $V=1 / 6 \Pi a b^{2}$, where $a$ represents the length and $b$ represents the width.

\section{HE staining}

To observe general histological pathology of tumor tissue, HE staining was used. Briefly, tumor tissues were dehydrated by gradient concentration of ethanol, vitrificated by dimethylbenzene, and then embedded in paraffin. Subsequently, $5 \mu \mathrm{m}$ sections were cut in a microtome. Then, sections were deparaffinized in dimethylbenzene $(3 \times 5$ minutes $)$ and rehydrated with contiguous 3-minute washes in 100\%, $95 \%, 90 \%$, and $85 \%$ ethanol. Then, they were stained 
with hematoxylin for 2 minutes, rinsed with tap water for 1 minute, rinsed with $1 \%$ hydrochloric acid in $75 \%$ ethanol for 20 seconds, rinsed with tap water for 1 minute, stained with eosin for 1 minute, and washed with tap water. The slides were dehydrated with $85 \%, 95 \%$, and $100 \%$ ethanol, followed by vitrification with xylene $(3 \times 5$ minutes $)$. Then, the slides were cover-slipped with neutral balsam. The images were collected by a conventional light microscope in five visual fields per section at $\times 100$ magnification under a bright-field view.

\section{Ultrastructure of tumor cells under transmission electron microscope}

The tumor tissues from the nude mice were fixed with $4 \%$ glutaraldehyde for 2 hours, and then post-fixed with 1\% osmium tetroxide for 2 hours. After pervaporation dehydration of acetone, tumor tissues were incised into ultrathin slices with thickness of 50-80 nm, and the ultrastructure of tumor cells was observed under the transmission electron microscope.

\section{Statistical analysis}

All data were obtained from at least three independent experiments and were expressed as mean \pm standard deviation. Multiple comparisons were analyzed by one-way analysis of variance. $P<0.05$ was considered statistically significant. All statistical analyses were performed and graphs were generated with GraphPad Prism v5.0 (GraphPad Prism Software, Inc., La Jolla, CA, USA).

\section{Results}

\section{The expression of IL- 2 and IFN- $\beta$}

\section{fusion gene}

In order to examine the antitumor activity of IL-2 and IFN- $\beta$ fusion protein, IL- 2 and IFN- $\beta$ fusion gene was inserted into the pEGFP-C1 (Clotech) and pcDNA3.1A. pEGFP-IL-2/IFN- $\beta$ and pcDNA3.1A-IL-2/IFN- $\beta$ were then transfected into Lovo cells. An $\sim 50 \mathrm{kDa}$ band was detected in the cells transfected with pcDNA3.1A-IL-2/IFN- $\beta$ using anti-IFN- $\beta$ antibody, while 15 and $25 \mathrm{kDa}$ bands were detected in cells transfected with pcDNA3.1A-IL-2 and pcDNA3.1A-IFN- $\beta$, respectively (Figure 1A). Furthermore, the fusion protein secreted by pcDNA3.1A-IL-2/IFN- $\beta$ was detected by ELISA using anti-IFN- $\beta$ or anti-IL-2 antibody (Figure 1B). Compared with pcDNA3.1A, the expression of IL-2 was significantly increased in pcDNA3.1A-IL-2 $(P<0.01)$ and pcDNA3.1A-IL-2/IFN- $\beta(P<0.05)$. Moreover, the expression of IFN- $\beta$ was significantly increased in
pcDNA3.1A-IFN- $\beta(P<0.01)$ and pcDNA3.1A-IL-2/IFN- $\beta$ $(P<0.05)$. As shown in Figure $1 \mathrm{C}$, the GFP exhibited a whole-cell location, whereas pEGFP-IL-2/IFN- $\beta$, a fusion protein including IL-2, IFN- $\beta$, and GFP, was mainly located in the cytoplasm of Lovo cells.

\section{Effects of pcDNA3. IA-CEA-IL-2/IFN- $\beta$ on cell morphology and apoptosis}

As CEA is a well-characterized tumor-associated antigen and expressed in most colon cancer cells, CEA promoter was inserted into the pcDNA3.1A-IL-2/IFN- $\beta$ to generate the plasmid pcDNA3.1A-CEA-IL-2/IFN- $\beta$. The expression of IL-2/IFN- $\beta$ fusion protein was under the control of CEA promoter. Then, pcDNA3.1A-CEA-IL-2/IFN- $\beta$ was transfected into Lovo cells, and the fusion protein was detected by indirect immunofluorescence using IL-2 antibody at 24 (Figure 2A) or 48 hours (Figure 2B) post-transfection. The fusion protein expressed by pcDNA3.1A-CEA-IL-2/ IFN- $\beta$ exhibited the same subcellular distribution as IL-2/IFN- $\beta$-GFP which was mainly located in the cytoplasm. However, the fusion protein could hardly be detected in HeLa cells because CEA was not expressed in HeLa cells (data not shown), which suggested that the expression of pcDNA3.1A-CEA-IL-2/IFN- $\beta$ was regulated by CEA promoter. Interestingly, morphology change was observed in the Lovo cells at 48 hours post-transfection with pcDNA3.1A-CEA-IL-2/IFN- $\beta$ (Figure 2B). The cells became round with smaller nuclei, implying that apoptosis was induced by IL- $2 /$ IFN- $\beta$ fusion protein.

Furthermore, flow cytometry assays were performed to identify the effects of pcDNA3.1A-CEA-IL-2/IFN- $\beta$ on apoptosis in Lovo cells (Figure 2C and D). Compared with the pcDNA3.1A control group, an obvious peak for apoptosis (blue) was found in cells transfected with pcDNA3.1A-CEAIL-2/IFN- $\beta$ (Figure 2C and D).

\section{Combined gene therapy attenuates tumor growth}

Nude mouse transplantation tumor model was used to evaluate the antitumor activity of pcDNA3.1A-CEA-IL-2/IFN- $\beta$ in vivo. Tumor formation was observed in all nude mice injected with $1 \times 10^{7}$ Lovo cells for $\sim 10$ days (Figure $3 \mathrm{~A}$ and B), suggesting a tumor formation rate of $100 \%$. The tumor size and weight were examined after gene therapy for 14 days. As shown in Figure 3C and D, injection with IL-2/IFN- $\beta$ expression plasmids led to significant decreases in tumor size and weight as compared with control group $(P<0.05)$. Furthermore, the antitumor effect via tail-vein injection seemed more 

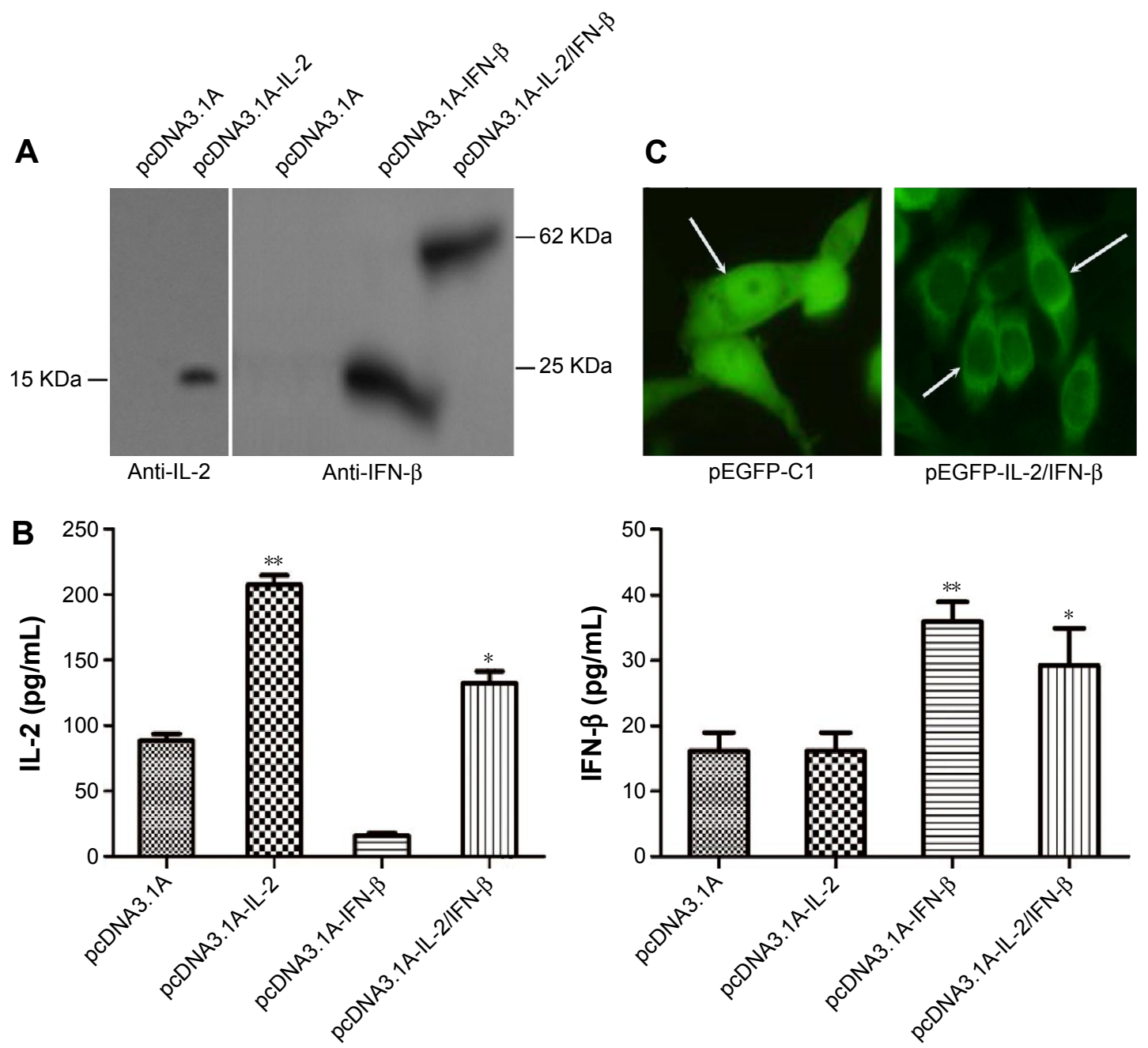

Figure I The expression of IL-2 and IFN- $\beta$ fusion protein in Lovo cells.

Notes: (A) The expression of pcDNA3. IA-IL-2, pcDNA3. IA-IFN- $\beta$, and pcDNA3. IA-IL-2/IFN- $\beta$ was detected by Western blot analysis in Lovo cells using specific antibodies. (B) ELISA was performed to detect the IL-2/IFN- $\beta$ fusion protein levels in supernatants of medium. Column: mean $(n=3)$; bar: $S D$. $* P<0.05$ vs $p c D N A 3$.IA; $* * P<0.0$ I vs pcDNA3.IA. (C) Subcellular distribution of IL-2/IFN- $\beta$-GFP and GFP in Lovo cells. Arrows represent the location of GFP. The control GFP was expressed in both the cytoplasm and nucleus, while IL-2/IFN- $\beta$-GFP fusion protein was mainly located in the cytoplasm of Lovo cells.

Abbreviations: IL-2, interleukin-2; IFN- $\beta$, interferon- $\beta$; ELISA, enzyme-linked immunosorbent assay; SD, standard deviation.

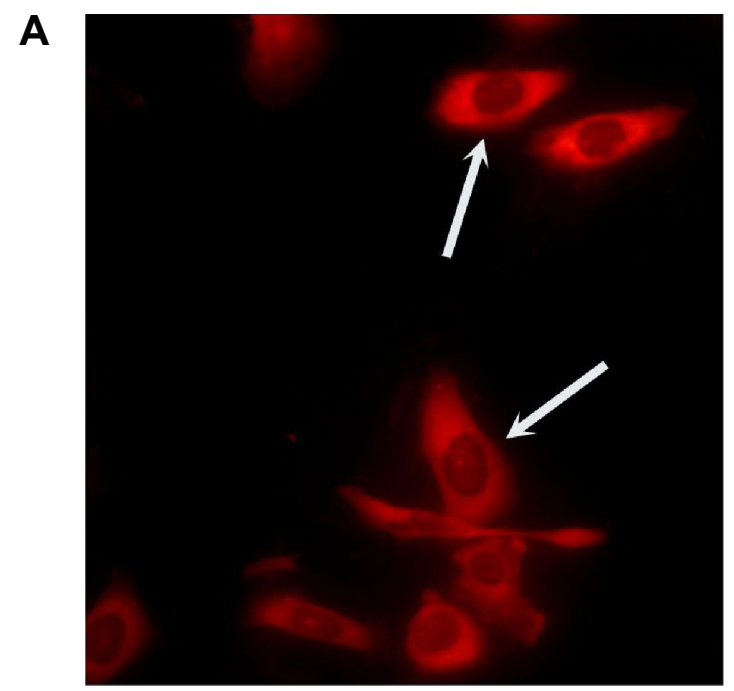

B

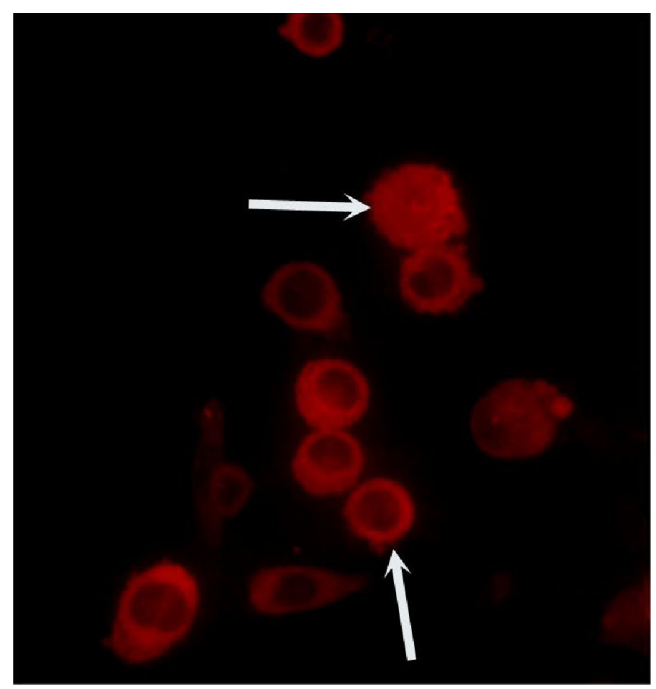

Figure 2 (Continued) 

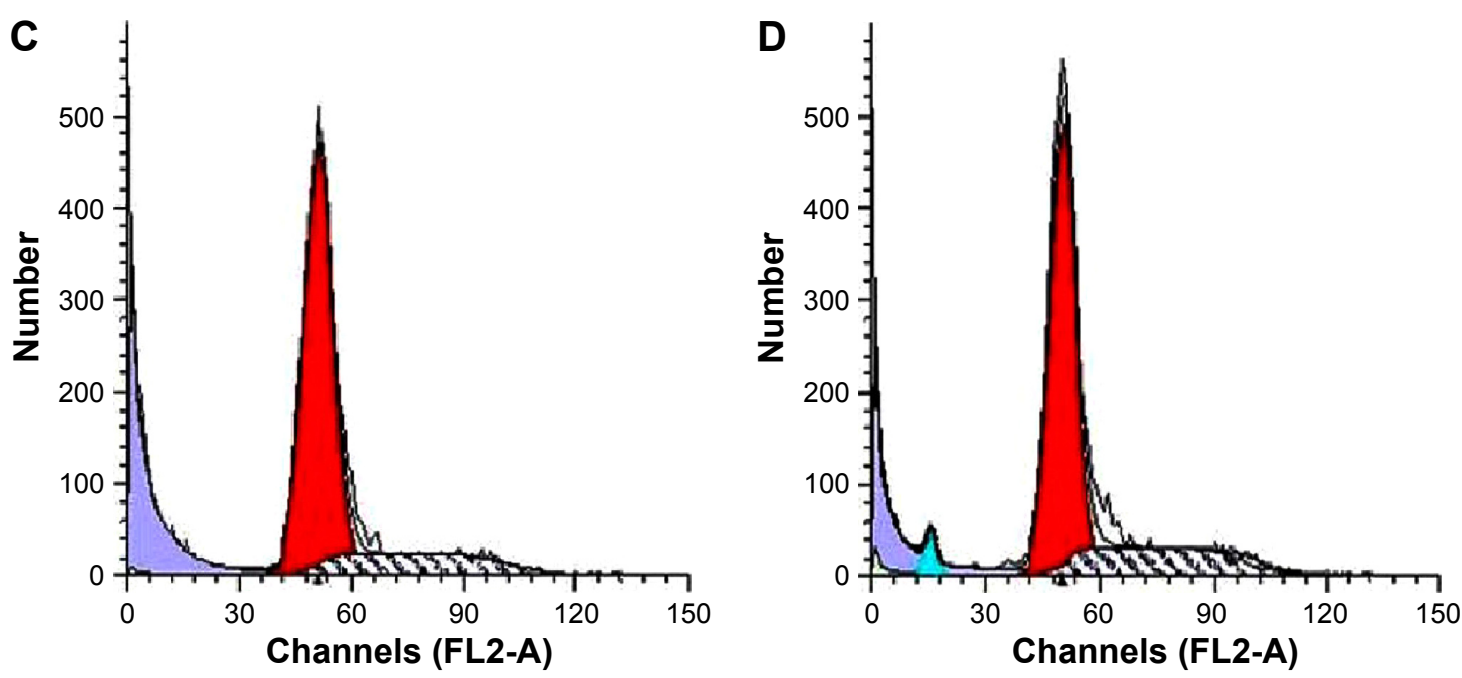

$\square$ Debris $\square$ Aggregates $\square$ Apoptosis $\square$ Dip G1 $\square$ Dip G2 $\Delta$ Dip S

Figure 2 Transfection of pcDNA3. IA-CEA-IL-2/IFN- $\beta$ led to apoptosis of Lovo cells.

Notes: After transfection of pcDNA3.IA-CEA-IL-2/IFN- $\beta$ into Lovo cells for (A) 24 and (B) 48 hours, immunofluorescence was performed to detect IL-2/IFN- $\beta$ fusion protein using anti-IL-2 antibody. Arrows represent the typical location of the fusion protein expressed by pcDNA3. IA-CEA-IL-2/IFN- $\beta$. Flow cytometry assay was performed to detect the cell apoptosis in Lovo cells after they were transfected with (C) pcDNA3.IA and (D) pcDNA3.IA-CEA-IL-2/IFN- $\beta$.

Abbreviations: IL-2, interleukin-2; IFN- $\beta$, interferon- $\beta$; Dip, diploid.

A
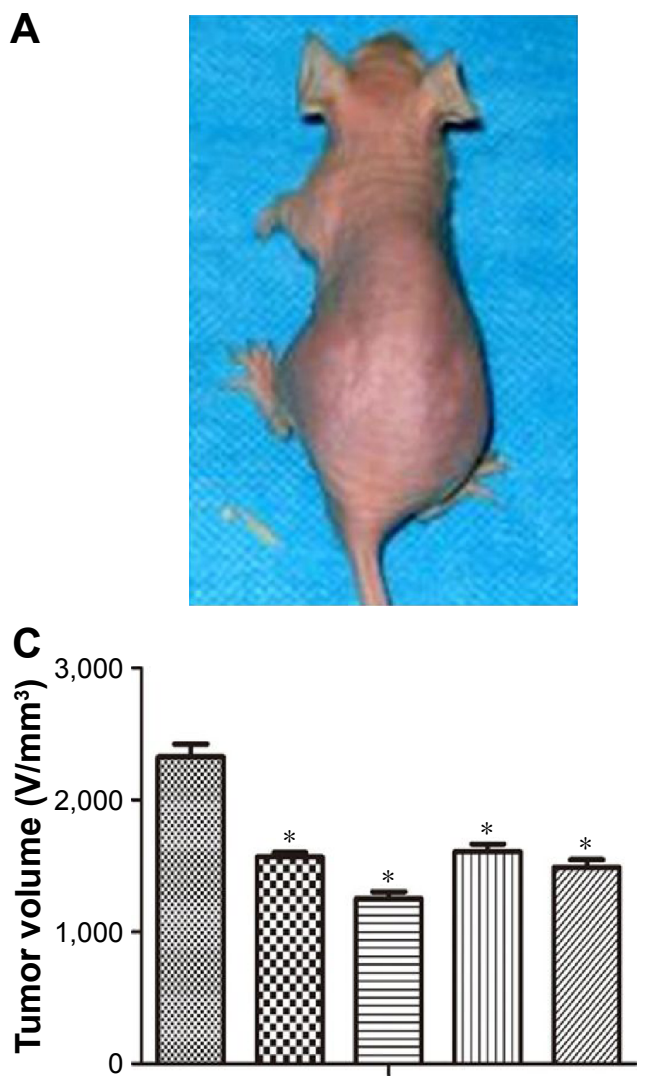

B

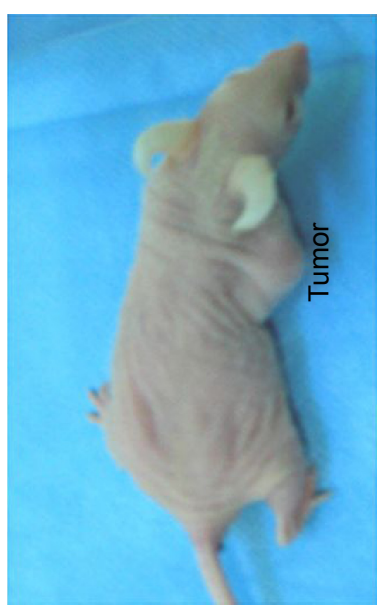

D

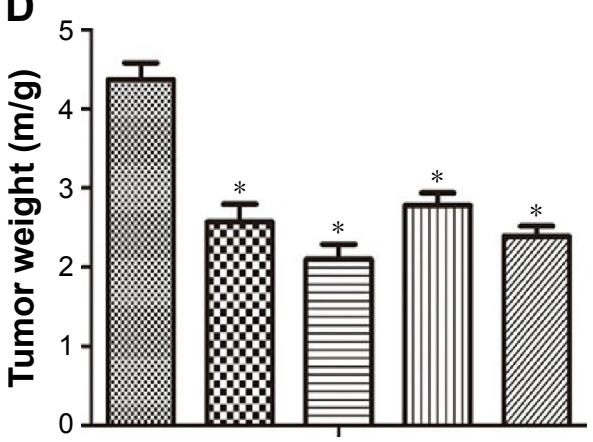

Group $1 \quad \mathbf{B}$ Group 2 目 Group 3 四 Group 4 Group 5

Figure 3 The plasmid pcDNA3.IA-CEA-IL-2/IFN- $\beta$ exhibited significant antitumor activity in nude mice tumor model.

Notes: Nude mouse were injected with (A) DMEM or (B) I $\times 10^{7}$ Lovo cells for 10 days. The (C) volume and (D) weight of tumor in each group were evaluated. Group I, liposomes + pcDNA3.IA by intratumor injection ( $n=5)$; Group 2, liposomes + pcDNA3.IA-IL-2/IFN- $\beta$ plasmid by intratumor injection ( $n=5$ ); Group 3, liposomes + pcDNA3.IA-IL-2/IFN- $\beta$ plasmid by tail intravenous injection $(n=5)$; Group 4, liposomes + pcDNA3.IA-CEA-IL-2/IFN- $\beta$ plasmid by intratumor injection ( $n=5$ ); Group 5, liposomes + pcDNA3. IA-CEA-IL-2/IFN- $\beta$ plasmid by tail intravenous injection $(n=5)$. Column: mean $(n=5)$; bar: SD. $* P<0.05$ vs $G$ roup I.

Abbreviations: DMEM, Dulbecco's Modified Eagle's Medium; SD, standard deviation. 
obvious than that via intratumor injection, which suggested the obvious targeting effect of CEA promoter. However, there was no significant difference in the antitumor effect between pcDNA3.1A-IL-2/IFN- $\beta$ and pcDNA3.1A-CEA-IL-2/IFN- $\beta$ $(P>0.05$; Figure $3 \mathrm{C}$ and $\mathrm{D})$.

\section{Combined gene therapy enhances tumor cells apoptosis}

To further investigate the antitumor effect of IL-2/IFN- $\beta$ fusion protein, flow cytometry assays were carried out to examine the apoptosis of tumor cells in nude mice model (Figure 4A). The results showed that almost no apoptosis cells were detected in the control group, while injection of pcDNA3.1-CEA-IL-2/IFN- $\beta$ in tumor induced an apoptosis rate of $8 \%$. Moreover, tail-vein injection with pcDNA3.1-CEA-IL-2/IFN- $\beta$ induced a higher apoptosis rate of $10 \%$.

The ultrastructure of tumor cells from each group was also analyzed using a transmission electron microscope. The control group exhibited big nucleus, evenly distributed nucleus mass, and abundant rough endoplasmic reticulum and mitochondria in the cytoplasm. In the nude mice injected with IL-2/IFN- $\beta$ in tumor, the cells showed typical apoptosis morphological changes, including narrow nuclei, chromatin agglutination, cell cavitation, and the formation of apoptotic bodies (Figure 4B). In addition, the DNA ladder electrophoresis was also performed to examine the apoptosis of tumor cells. The DNA ladder was observed, and DNA in nucleosomes was degraded into multiple 180-200 bp oligonucleotides in the groups injected with pcDNA3.1CEA-IL-2/IFN- $\beta$ (Figure 4C).

\section{Pathological changes of tumor tissue after combined gene therapy}

As shown in Figure 5A, tumor cells were significantly decreased in gene therapy groups via intratumor injection and tail intravenous injection compared with those in control group. Meanwhile, a degenerative change, a significant decrease of mitoses, and a large number of vacuolar cells were observed in tumor tissue injected with IL-2/IFN- $\beta$. The HE staining results also indicated that cell pycnosis and cavitation appeared in the tumor tissues in the groups injected with pcDNA3.1-CEA-IL-2/IFN- $\beta$ in tumor or in tail vein (Figure 5B and C).
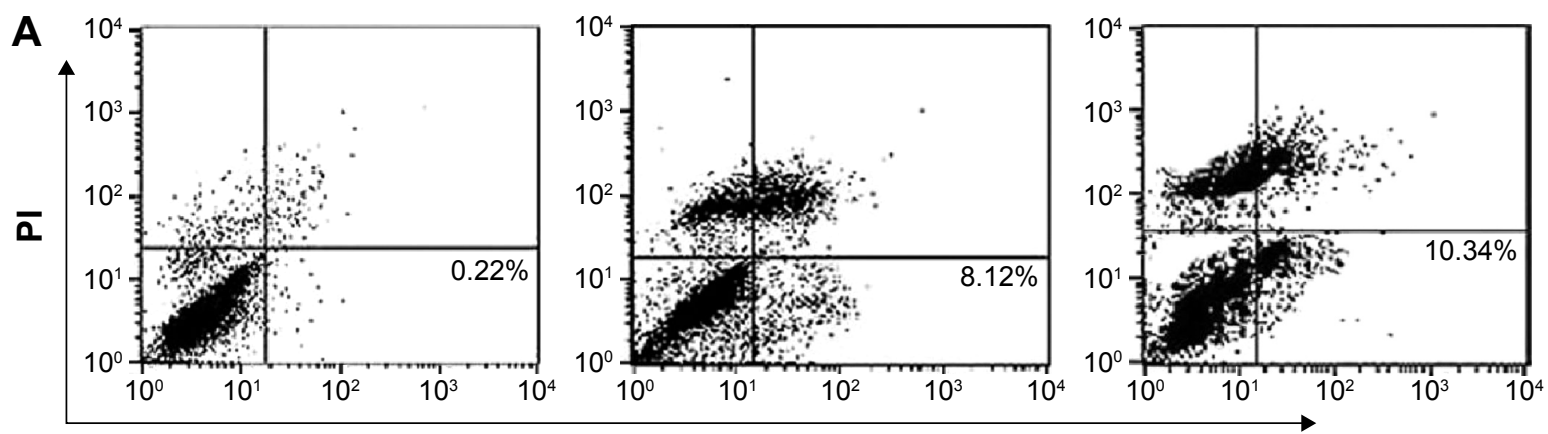

\section{Annexin V-FITC}

B
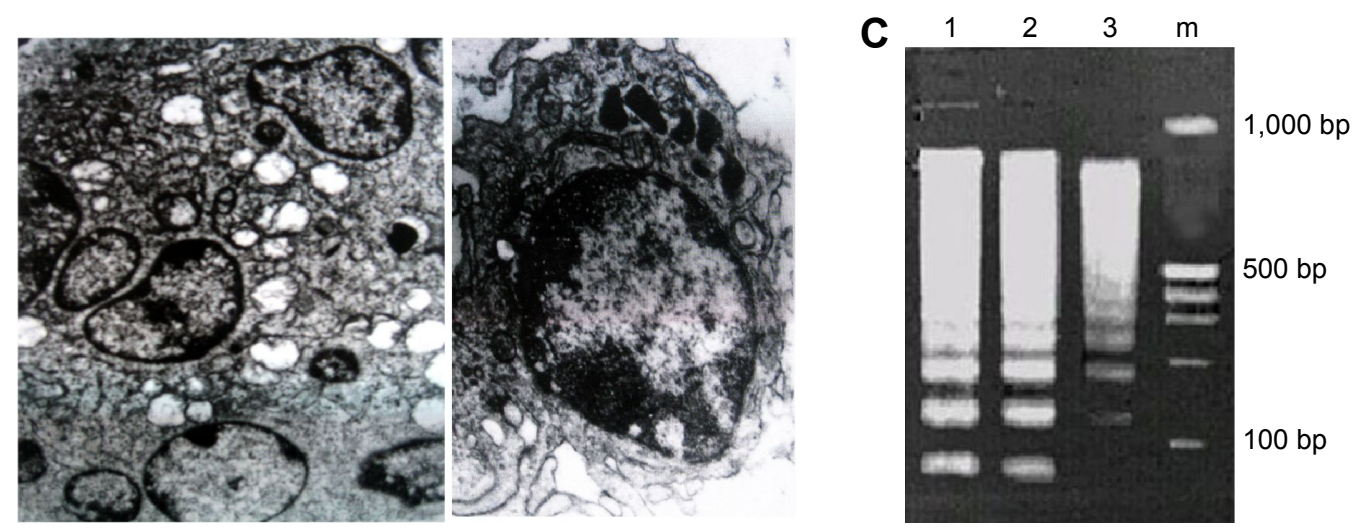

Figure 4 Transfection of pcDNA3.IA-CEA-IL-2/IFN- $\beta$ induced apoptosis in the tumor.

Notes: (A) Flow cytometry was carried out to examine the apoptosis of tumor cells in nude mice injected with pcDNA3.IA in tumor (left), pcDNA3.IA-IL-2/IFN- $\beta$ in tumor (middle), or pcDNA3.IA-CEA-IL-2/IFN- $\beta$ via the tail vein (right). (B) Analysis of the ultrastructure of tumor in nude mice injected with pcDNA3.IA-IL-2/IFN- $\beta$ in tumor by transmission electron microscope. (C) DNA ladder was detected in the DNA extracted from the tumor of nude mice injected with pcDNA3.I-CEA-IL-2/IFN- $\beta$ via the tail vein. The "m" means TaKaRa DNA marker.

Abbreviations: PI, propidium iodide; FITC, fluorescein isothiocyanate. 

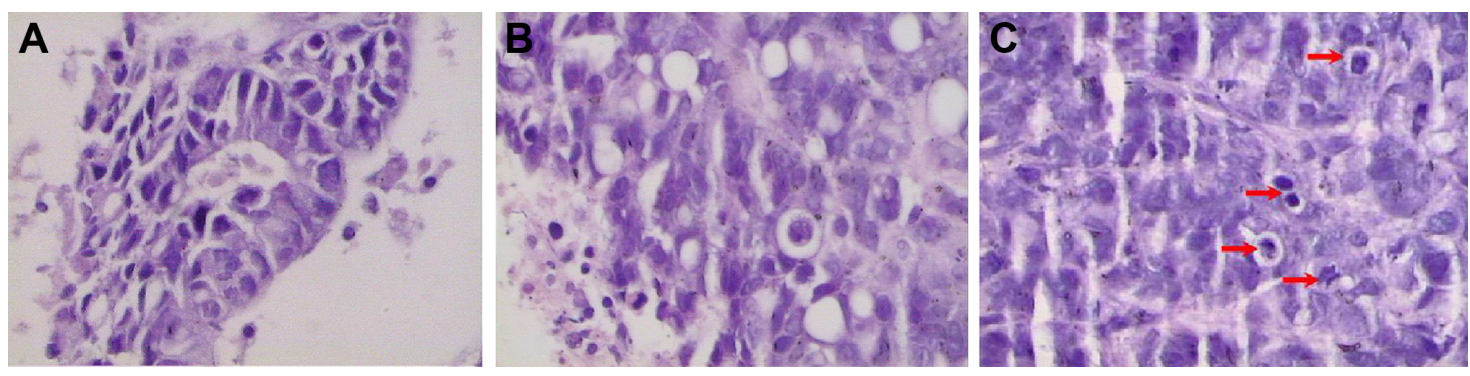

Figure 5 Pathological changes of tumor tissue after combined gene therapy.

Notes: Hematoxylin and eosin staining was performed to assess the pathological changes of tumor tissues from nude mice injected with (A) pcDNA3.IA in tumor, (B) pcDNA3.IA-CEA-IL-2/IFN- $\beta$ in tumor, and (C) pcDNA3. IA-CEA-IL-2/IFN- $\beta$ in tail vein. Arrows show the cell pycnosis and cavitation in tumor tissues.

\section{Discussion}

$\mathrm{CRC}$ is one of the most aggressive types of cancers and is relatively resistant to chemotherapy. ${ }^{21}$ Currently, growing evidences have suggested that therapeutic benefits of combinatorial gene therapy are synergistic via activating different immune pathways. ${ }^{22,23}$ In this study, with the aim to evaluate the antitumor effects of combined IL-2/IFN- $\beta$-based gene therapy, we derived a highly potent CEA promoter which was capable of targeting special genes. First of all, an animal model of CRC was induced via injection of Lovo cells to nude mice. Then, the injection of IL-2/IFN- $\beta$ was found to inhibit the tumor growth and induce cell apoptosis in vivo. These results suggest that IL-2/IFN- $\beta$ synergistically has a critical role in the anti-CRC treatment.

As we all know, IL-2 and IFN- $\beta$ both have underlying antitumor effects not only in animal research but also in clinical trials. ${ }^{24,25}$ Therefore, the strong synergistic effect of the IL-2 and IFN- $\beta$ was demonstrated, and our results were in accordance with previous studies. In our study, tumor growth was significantly inhibited in combined IL-2/IFN- $\beta$ gene therapy group relative to that of control group in a murine $\mathrm{CRC}$ model. Additionally, no mice in the group that received the combinatorial delivery of IFN- $\beta$ and IL- 2 had died by the end of experiment after Lovo cells implantation. Our results indicate that combined IL-2/IFN- $\beta$ gene therapy is an effective antitumor strategy, which not only suppresses tumor growth but also prevents tumor-related death. The synergistic effect can be interpreted by some mechanisms. On the one hand, IFN- $\beta$ inhibits tumor vascularization via reducing the expression level of angiogenic factor. ${ }^{26} \mathrm{On}$ the other hand, IFN can enhance the ability of natural killer (NK) cells killing tumor cells through prompting the maturation and activation of NK cells. ${ }^{14,16}$ To our knowledge, IL-2 induced IFN production by stimulating cytotoxic T lymphocytes cells and NK cells, which further mediate the activation of monocyte/macrophage and production of tumor necrosis factor. Then, tumor necrosis factor- $\alpha$ induced necrosis of tumor cells via destructing the vasculature of tumors and caused apoptosis.

A body of evidence indicates that it may be effective to induce the apoptotic processes to prevent tumor formation, recurrence, and metastasis. ${ }^{27,28}$ In the present study, treatment with IL-2/ IFN- $\beta$ following Lovo cells transfection induced a significant apoptosis examined by flow cytometry, DNA ladder electrophoresis, HE staining, and transmission electron microscopy. These results demonstrate that IL-2/IFN- $\beta$ gene therapy induces the occurrence of apoptosis to further inhibit the tumor growth.

Although gene therapy has made some progress, specific gene delivery is a major shortcoming that limits its use in clinic. ${ }^{29}$ Fortunately, CEA promoter holds potential to solve this problem. A former study has demonstrated that Cre/loxP system significantly improves the selective expression of specific gene by means of CEA promoter. ${ }^{30}$ Thus, in the current study, we constructed the CEA as the promoter to examine the anti-CRC efficacy of combinatorial delivery of IFN- $\beta$ and IL-2. Subsequently, we evaluated CEA promoter activity. In the current study, IL-2/IFN- $\beta$-based gene therapy via tail-vein injection resulted in much greater reduction in both tumor size and tumor weight than that via intratumor injection. Moreover, there was no significant difference in the antitumor effect between pcDNA3.1A-IL-2/IFN- $\beta$ and pcDNA3.1A-CEA-IL-2/IFN- $\beta$. This finding can be explained as strict regulation of the specificity of the CEA promoter.

In conclusion, these results suggest that the combination of IL-2/IFN- $\beta$ exerts strong and synergistic antitumor effects by inducing apoptosis and activating IL-2/IFN- $\beta$-related immune responses..$^{31}$ Also, the fusion gene of IL-2/IFN- $\beta$ was recombined via linker to increase the transduction efficiency, and CEA promoter could be used to enhance the targeting specificity of IL-2/IFN- $\beta$. To a certain degree, our results will provide the theoretical basis of IFN- $\beta$ and IL- 2 combination gene therapy to develop clinic therapy for CRC. However, the efficacy of the combination of IL-2 and IFN- $\beta$ in CRC treatment should also be investigated in clinic in future. 


\section{Disclosure}

The authors report no conflicts of interest in this work.

\section{References}

1. Stewart BW, Wild C. World Cancer Report 2014. Geneva: World Health Organization; 2014.

2. Szepeshazi K, Schally A, Groot K, et al. Antagonists of growth hormone-releasing hormone (gh-rh) inhibit igf-ii production and growth of ht-29 human colon cancers. Br J Cancer. 2000;82:1724.

3. Lorenz M, Staib-Sebler E, Hochmuth K, et al. Surgical resection of liver metastases of colorectal carcinoma: short and long-term results. Semin Oncol. 2000;27:112-119.

4. Terzić J, Grivennikov S, Karin E, Karin M. Inflammation and colon cancer. Gastroenterology. 2010;138:2101.e2105-2114.e5.

5. Guo Y, Xu F, Lu T, Duan Z, Zhang Z. Interleukin-6 signaling pathway in targeted therapy for cancer. Cancer Treat Rev. 2012;38:904-910.

6. Merchant K, Kumi-Diaka J, Rathinavelu A, et al. Genistein modulation of immune-associated genes in Incap prostate cancer cell line. Genesis. 2012; 11:15.

7. Krastev Z, Koltchakov V, Tomova R, et al. Locoregional il-2 low dose applications for gastrointestinal tumors. World J Gastroenterol. 2005; 11:5525.

8. Kusnierczyk H, Pajtasz-Piasecka E, Koten J-W, et al. Further development of local il-2 therapy of cancer: multiple versus single il-2 treatment of transplanted murine colon carcinoma. Cancer Immunol Immunother. 2004;53:445-452.

9. Lissoni P, Brivio F, Fumagalli L, Di Fede G, Brera G. Enhancement of the efficacy of chemotherapy with oxaliplatin plus 5-fluorouracil by pretreatment with il-2 subcutaneous immunotherapy in metastatic colorectal cancer patients with lymphocytopenia prior to therapy. In Vivo. 2005; 19:1077-1080.

10. Correale P, Cusi MG, Tsang KY, et al. Chemo-immunotherapy of metastatic colorectal carcinoma with gemcitabine plus folfox 4 followed by subcutaneous granulocyte macrophage colony-stimulating factor and interleukin-2 induces strong immunologic and antitumor activity in metastatic colon cancer patients. J Clin Oncol. 2005;23:8950-8958.

11. Sobol RE, Shawler DL, Carson C, et al. Interleukin 2 gene therapy of colorectal carcinoma with autologous irradiated tumor cells and genetically engineered fibroblasts: a phase i study. Clin Cancer Res. 1999;5: 2359-2365.

12. Taniguchi F, Yamagishi H, Fujiwara H, et al. Systemic administration of ril-12 synergistically enhances the therapeutic effect of a tnf genetransduced cancer vaccine. Gene Ther. 1998;5:1677-1684.

13. Gunji Y, Tasaki K, Tagawa M, et al. Inhibition of peritoneal dissemination of murine colon carcinoma cells by administrating retrovirus harboring il-2 gene. Cancer Gene Ther. 1998;5:339-343.

14. Qin XQ, Tao N, Dergay A, et al. Interferon-beta gene therapy inhibits tumor formation and causes regression of established tumors in immunedeficient mice. Proc Natl Acad Sci U S A. 1998;95:14411-14416.

15. Brunda MJ, Bellantoni D, Sulich V. In vivo anti-tumor activity of combinations of interferon alpha and interleukin-2 in a murine model. Correlation of efficacy with the induction of cytotoxic cells resembling natural killer cells. Int J Cancer. 1987;40:365-371.
16. Hiroishi K, Tuting T, Lotze MT. Ifn-alpha-expressing tumor cells enhance generation and promote survival of tumor-specific ctls. J Immunol. 2000;164:567-572.

17. Mendiratta SK, Quezada A, Matar M, et al. Combination of interleukin 12 and interferon alpha gene therapy induces a synergistic antitumor response against colon and renal cell carcinoma. Hum Gene Ther. 2000; 11:1851-1862.

18. Higashi K, Hazama S, Araki A, et al. A novel cancer vaccine strategy with combined il-18 and hsv-tk gene therapy driven by the htert promoter in a murine colorectal cancer model. Int J Oncol. 2014;45:1412-1420.

19. Schrewe H, Thompson J, Bona M, et al. Cloning of the complete gene for carcinoembryonic antigen: analysis of its promoter indicates a region conveying cell type-specific expression. Mol Cell Biol. 1990;10: $2738-2748$.

20. Konishi F, Maeda H, Yamanishi Y, et al. Transcriptionally targeted in vivo gene therapy for carcinoembrionic antigen-producing adenocarcinoma. Hiroshima J Med Sci. 1999;48:79-89.

21. Csiszár A, Szentes T, Haraszti B, et al. The pattern of cytokine gene expression in human colorectal carcinoma. Pathol Oncol Res. 2004 10:109-116.

22. Chaudhry UI, Kingham TP, Plitas G, et al. Combined stimulation with interleukin-18 and cpg induces murine natural killer dendritic cells to produce ifn-gamma and inhibit tumor growth. Cancer Res. 2006; 66:10497-10504.

23. Stagg J, Wu JH, Bouganim N, Galipeau J. Granulocyte-macrophage colony-stimulating factor and interleukin-2 fusion cdna for cancer gene immunotherapy. Cancer Res. 2004;64:8795-8799.

24. Caporale A, Brescia A, Galati G, et al. Locoregional il-2 therapy in the treatment of colon cancer. Cell-induced lesions of a murine model. Anticancer Res. 2007;27:985-989.

25. Ren C, Kumar S, Chanda D, et al. Cancer gene therapy using mesenchymal stem cells expressing interferon- $\beta$ in a mouse prostate cancer lung metastasis model. Gene Ther. 2008;15:1446-1453.

26. Singh RK, Gutman M, Bucana $C D$, et al. Interferons alpha and beta down-regulate the expression of basic fibroblast growth factor in human carcinomas. Proc Natl Acad Sci U S A. 1995;92:4562-4566.

27. Lorenz H, Herrmann M, Winkler T, Gaipl U, Kalden J. Role of apoptosis in autoimmunity. Apoptosis. 2000;5:443-449.

28. Schulze-Bergkamen H, Krammer PH. Apoptosis in cancer-implications for therapy. Semin Oncol. 2004;31:90-119.

29. Lan K-H, Kanai F, Shiratori Y, et al. In vivo selective gene expression and therapy mediated by adenoviral vectors for human carcinoembryonic antigen-producing gastric carcinoma. Cancer Res. 1997; 57:4279-4284.

30. Kijima T, Osaki T, Nishino K, et al. Application of the cre recombinase/ loxp system further enhances antitumor effects in cell type-specific gene therapy against carcinoembryonic antigen-producing cancer. Cancer Res. 1999;59:4906-4911.

31. Yi BR, Park MA, Lee HR, et al. Suppression of the growth of human colorectal cancer cells by therapeutic stem cells expressing cytosine deaminase and interferon-beta via their tumor-tropic effect in cellular and xenograft mouse models. Mol Oncol. 2013;7:543-554.
OncoTargets and Therapy

\section{Publish your work in this journal}

OncoTargets and Therapy is an international, peer-reviewed, open access journal focusing on the pathological basis of all cancers, potential targets for therapy and treatment protocols employed to improve the management of cancer patients. The journal also focuses on the impact of management programs and new therapeutic agents and protocols on

\section{Dovepress}

patient perspectives such as quality of life, adherence and satisfaction. The manuscript management system is completely online and includes a very quick and fair peer-review system, which is all easy to use. Visit http://www.dovepress.com/testimonials.php to read real quotes from published authors. 